\title{
Neonatal Screening for Hearing Loss Using OAE and Comparison it with Conventional Methods
}

\author{
Dr. Amit Kumar ${ }^{1}$, Dr. Binod Kumar Sinha ${ }^{2}$, Dr. Dheeraj Kumar ${ }^{3}$ \\ ${ }^{1}$ Senior Resident, Department of Otorhinolaryngology, Rajendra Institute of Medical Sciences, Ranchi. \\ ${ }^{2}$ Assistant Professor, Department of Otorhinolaryngology, Rajendra Institute of Medical Sciences, Ranchi. \\ ${ }^{3}$ Junior Resident, Department of Otorhinolaryngology, Rajendra Institute of Medical Sciences, Ranchi.
}

\begin{abstract}
:
Introduction: Communication is the essence of life. Speech, Language and Hearing are involved rigorously in any communication around us. Children Hearing loss in children constitutes a considerable handicap because it is an invisible disability \& compromises optimal development \& personnel achievement of a child. Screening is one of the most important methods for early diagnosis of hearing loss. Otoacoustic Emissions (OAE's) is an easy, cost effective and reliable method of testing of large number of infants for hearing loss.

Material and Method: The present study was a Pilot study conducted at the Rajendra Institute of Medical Sciences, Ranchi during the period one year, with a sample size of 100 including normal as well high risk babies. Babies were assessed by Conventional method, DPOAE and by ABR at five different Phases at different time intervals.

Results: The overall detection rate by DPOAE over Conventional methods was higher by $36 \% .197 .9 \%$ Vs 61.6\%) The Sensitivity of the Conventional methods has progressively increased from Phase-I to Phase-IV (from $20 \%$ to 90\%). Negative predictive value for Conventional methods was high at initial Phase and reduced at later Phases.

Conclusion: Hence the use of Distortion product Otoacoustic Emissions as initial screening test provides as easy, cost effective and quick method to detect infants with hearing loss. As it is less invasive and time consuming than BERA, DPOAE can be used as initial screening method for hearing loss in infants with BERA being reserved for infants that fail DPOAE screening.
\end{abstract}

Keywords: Infant; Distortion Product Otoacoustic Emissions; Brainstem Evoked Response Audiometry. Conventional Methods

\section{Introduction}

Congenital Sensorineural hearing impairment has been estimated $1.2-5.7$ / thousand live birth. ${ }^{1,2,3,4,5}$ The Prevalence of hearing loss in infant is more than twice that of other Screened new born disorders like hypothyroidism and phenyl ketonuria . In addition 50 per. Of children with moderate to propound congenital hearing loss exhibit no risk factor for hearing loss. ${ }^{67,8,9,10}$ Early detection followed by appropriate treatment provided the best choice maximizing the critical period of hearing to avail of resources to improve hearing and oral communication skills. On the other hand late deduction and treatment leaves the child with poor speech development and school achievement.

In most countries, newborn hearing screening programmes that screen only high-risk infants have been in existence for more than 20 years. However, this group of infants with hearing loss comprises only $50 \%$ of newborn population with hearing loss. Therefore, hearing screening programs that screened only high-risk neonates missed out 50\% of hearing-impaired newborns, who are from among infants without any risks factors. Also as hearing loss is an invisible disability it cannot be passively identified until the child fails to develop speech and language.

Hearing impairment in infants should be identified as early as possible to enable interventions to take full advantage of the plasticity of developing sensory system. Hearing integrity in the first 3-4 years of life, the 'critical period', is essential for acquisition of speech and language. Unfortunately, by the time hearing loss in infancy and early childhood is suspected, audiologically evaluated and appropriately managed two or more of these critical years have elapsed and the child has lost an enormous developmental advantage. The onus lies on modern physicians to innovate culturally acceptable ways of implementing Infant Hearing Screening programs.

Otoacoustic Emissions (OAE) reflect the status of the cochlea (outer hair cells).A probe microphone similar to that used in acoustic immitance measures the inaudible sounds reflected by vibratory motion in cochlea. OAE's are a byproduct of sensory outer hair cell transduction and are reflected as echoes into the external auditory canal. OAE's are preneural in origin and directly dependant on outer hair cell integrity.

Brainstem Evoked Response Audiometry (BERA) is an objective test of audiological function which measures activity from the auditory nerve up to the level of brainstem on stimulating with acoustic stimulus. It 
assesses the neural integrity of auditory pathway up to the brainstem. However it is an indirect measure of hearing acuity.

\section{Materials And Methodology}

The study was a Pilot study to screen for the incidence of hearing loss among the infants born in Rajendra Institute of medical science. Ranchi. The objective of the study was to screen the hospital born infants for the incidence of hearing impairment, using Distortion Product Otoacoustic Emissions (DPOEA) Vs clinically practiced Conventional methods, over a period of one year.

\section{Inclusion criteria:}

1. Neonates born at Rajendra Institute of Medical Science and occupying the bed for a minimum of five days.

2. It included both Normal and High risk neonates.

Exclusion criteria

1. Neonates suffering from critical illness were excluded from hearing

A proper history was taken from the parents about the normal course of pregnancy. Also history was taken to exclude any infection during pregnancy, history of use of ototoxic drugs, presence of family history of hearing loss. The following information of the infant was noted: gestational age, sex, maternal history, prenatal and maternal risk factors, and birth weight, APGAR score at 5 and 10 minutes and postnatal complications. APGAR score was recorded using colour, heart rate, respiration, reflex response and motor response. After otoscopic examination of the ears, screening was done. With the infant lying comfortably on the bed or the mother's lap, testing was carried out in a sound treated room.

Conventional Methods: Acoustic blink reflex - It is a blinking of the infant's eyes in response to a sudden sharp sound produced by snapping fingers, using a bell, beeper or other noise making device. Approximately one foot away from infant's ear, be sure you are not producing an airstream that may cause the infants to blink. After it is elicited several times with in a brief period the reflex disappear, a phenomenon known as habituation.

Distortion Product Otoacoustic Emission (DPOAE): Hearing screening examination was done using the instrument. DPOAE, the recording was done in a noise free room next to the maternal ward in natural sleep, no sedation is given.

\section{Results}

Statistical Analysis: The data was analyzed by using SPSS 20 software. The data is presented in percentages, rates and ratios. Chi square test was used to find the association between attributes.

Screening results \& Comparisons of phase-I: In Phase-I, the screening was done for 100 babies before 48 hours after birth. Only 3 (3\%) were detected by conventional method, while at the same phase when all the 100 were screened by DPOAE the detection was $15(13 \%)$. Another 12(12\%) babies were detected by DPOAE.

The comparison of outcome of conventional methods conducted on babies with gold standard DPOAE at PhaseI. Here only three babies responded for the conventional methods where as another 12 more babies were detected by DPOAE. True positives for Conventional method were $20 \%$ and false negative rate was $80 \%$. At Phase-I 15 have passed both methods. The sensitivity of the conventional methods stands at $20 \%$. The specificity of the same stands at $100 \%$. The positive predictive value (PPV) for the conventional methods stands at $100 \%$. The negative predictive value (NPV) for the conventional methods is at $87.6 \%$.

Table - I: Screening results of phase-I

\begin{tabular}{|c|c|c|c|}
\hline \multicolumn{4}{|c|}{ Phase-I $(<48$ hours of birth $)$} \\
\hline Method & Result & Number & Percentage \\
\hline \multirow{3}{*}{ Conventional method } & Passed & 3 & 3 \\
\cline { 2 - 4 } & Failed & 97 & 97 \\
\cline { 2 - 4 } & Total & 100 & 100 \\
\hline \multirow{2}{*}{ DPOAE } & Passed & 15 & 15 \\
\cline { 2 - 4 } & Referred & 85 & 85 \\
\cline { 2 - 4 } & Total & 100 & 100 \\
\hline
\end{tabular}

Table - II: Comparison of Conventional methods with DPOAE at Phase-I

\begin{tabular}{|l|l|l|l|l|}
\hline \multicolumn{2}{|c|}{} & \multicolumn{2}{|l|}{ Phase-I<48hours. DPOAE } & \multirow{2}{*}{ Total } \\
\cline { 3 - 4 } \multicolumn{2}{|c|}{} & Passed & Referred & \\
\hline \multirow{3}{*}{ Conventional methods. } & Passed & 3 & 0 & 3 \\
\cline { 2 - 5 } & $\%$ & $20 \%$ & 0 & $3 \%$ \\
\cline { 2 - 5 } & Referred & 12 & 85 & 97 \\
\hline
\end{tabular}


Neonatal Screening For Hearing Loss Using OAE And Comparison It With Conventional Methods

\begin{tabular}{|l|l|l|l|l|}
\hline & $\%$ & 80 & 100 & $97 \%$ \\
\hline \multirow{2}{*}{ Total } & 15 & 85 & 100 \\
\cline { 2 - 4 } & $100 \%$ & $100 \%$ & $100 \%$ \\
\hline
\end{tabular}

Sensitivity $=20 \%$. Specificity $=100 \%$. Positive predictive value $=100 \%$. Negative predictive value $=87.6 \%$

Screening results \& Comparisons of phase-II: At Phase-II, 24 hours later to Phase-I, of the 85 babies referred from Phase-I, the conventional method detected 8 (9.4\%) while at the same Phase-II, DPOAE was able to detect $18(21.8 \%)$. Another 10(12\%) babies were detected by DPOAE.

The comparison of outcome of Conventional methods conducted on babies with gold standard DPOAE at Phase-II. Here out of 85 babies referred from Phase-I, 8 babies passed exclusively conventional methods and another 10 were passed DPOAE. The true Positive value for Conventional method is $44 \%$ and false negative rate $56 \%$. The sensitivity of the conventional methods stands at $44 \%$, specificity at $100 \%$, the PPV at $100 \%$ and NPV at $87 \%$.

Table - III: Screening results of phase-II

\begin{tabular}{|c|c|c|c|}
\hline \multicolumn{4}{|c|}{ Phase-II (next day of Phase-I) (N= 85) } \\
\hline Method & Result & Number & Percentage \\
\hline Conventional method & Passed & 8 & 9.4 \\
\cline { 2 - 4 } & Failed & 77 & 90.6 \\
\cline { 2 - 4 } & Total & 85 & 100 \\
\hline \multirow{3}{*}{ DPOAE } & Passed & 18 & 21.8 \\
\cline { 2 - 4 } & Referred & 67 & 78.8 \\
\cline { 2 - 4 } & Total & 85 & 100 \\
\hline
\end{tabular}

Table - IV: Comparison of Conventional methods with DPOAE at Phase-II

\begin{tabular}{|l|l|l|l|l|}
\hline \multicolumn{2}{|c|}{} & $\begin{array}{l}\text { Phase-II Next day of Phase-I. } \\
\text { DPOAE }\end{array}$ & Total \\
\cline { 3 - 5 } & Passed & Referred & \\
\hline \multirow{3}{*}{ Conventional methods. } & Passed & 8 & 0 & 8 \\
\cline { 2 - 5 } & $\%$ & $44 \%$ & 0 & $9.4 \%$ \\
\cline { 2 - 5 } & Referred & 10 & 67 & 97 \\
\cline { 2 - 5 } & $\%$ & $56 \%$ & $100 \%$ & $90.6 \%$ \\
\hline \multirow{2}{*}{ Total } & 18 & 67 & $100 \%$ \\
\cline { 2 - 4 } & $100 \%$ & $100 \%$ & $10 \%$ \\
\hline
\end{tabular}

Sensitivity $=44 \%$. Specificity $=100 \%$. Positive predictive value $=100 \%$. Negative predictive value $=87 \%$

Screening results \& Comparisons of phase-III: In Phase-III, before getting discharged from hospital. Of the 67 babies referred from Phase-II, 30 (44.8\%) were detected by conventional method. And at same phase by using DPOAE on all the 67 babies the detection was higher at $45(67.2 \%)$. Another $15(33.3 \%)$ babies were detected by DPOAE.

The comparison of outcome of Conventional methods conducted on babies with gold standard DPOAE at Phase-III. Here out of 67 babies referred from Phase-II, 30 have passed exclusively by Conventional methods and another 15 babies were passed by DPOAE. The true positive value for conventional methods is $66.7 \%$ and false negative rate has reduced to $33.3 \%$. The sensitivity of Conventional methods is at $66.7 \%$, specificity at $100 \%$, the PPV at $100 \%$ and NPV at $59.6 \%$.

Table - V: Screening results of phase-III

\begin{tabular}{|c|c|c|c|}
\hline \multicolumn{3}{|c|}{ PHASE-III (Before discharge ) (N= 67) } \\
\hline Method & Result & Number & Percentage \\
\hline Conventional method & Passed & 30 & $44.8 \%$ \\
\cline { 2 - 4 } & Failed & 37 & $55.2 \%$ \\
\cline { 2 - 4 } & Total & 67 & $100 \%$ \\
\hline \multirow{3}{*}{ DPOAE } & Passed & 45 & $67.2 \%$ \\
\cline { 2 - 4 } & Referred & 22 & $32.8 \%$ \\
\cline { 2 - 4 } & Total & 67 & $100 \%$ \\
\hline
\end{tabular}

Table - VI: Comparison of Conventional methods with DPOAE at Phase-III

\begin{tabular}{|l|l|l|l|l|}
\hline \multicolumn{2}{|c|}{} & \multicolumn{2}{|l|}{ Phase-III DPOAE } & \multirow{2}{*}{ Total } \\
\cline { 3 - 5 } \multicolumn{2}{|c|}{} & Passed & Referred & \\
\hline \multirow{3}{*}{ Conventional methods. } & Passed & 30 & 0 & 30 \\
\cline { 2 - 5 } & $\%$ & $66.7 \%$ & 0 & $44.8 \%$ \\
\cline { 2 - 5 } & Referred & 15 & 22 & 37 \\
\cline { 2 - 5 } & $\%$ & $33.3 \%$ & $100 \%$ & $55.2 \%$ \\
\hline
\end{tabular}




\begin{tabular}{|l|l|l|l|}
\hline \multirow{2}{*}{ Total } & 45 & 22 & 67 \\
\cline { 2 - 4 } & $100 \%$ & $100 \%$ & $100 \%$ \\
\hline
\end{tabular}

Sensitivity $=66.7 \%$. Specificity $=100 \%$. Positive predictive value $=100 \%$. Negetive predictive value $=59.6 \%$

Screening results \& Comparisons of phase-IV: In Phase-IV, forty five days from Phase-I. Of the 21 babies referred from Phase-III, conventional method detected 18 (85.7\%) babies while at same phase the DPOAE was able to detect 20 (95.2\%). Another Two babies (10\%) was detected by DPOAE. One baby missed the evaluation at this phase.

The comparison of outcome of Conventional methods conducted on babies with gold standard DPOAE at Phase-IV. Here out of 21 babies referred from Phase-III, 18 have passed exclusively by Conventional methods and another 2 baby was passed by DPOAE. The true positive value for conventional methods is $90 \%$ and false negative rate has reduced to $20 \%$. The sensitivity of Conventional methods is at $90 \%$, specificity at $100 \%$, the PPV at $100 \%$ and NPV at $33.3 \%$.

Table - VII: Screening results of phase-IV

\begin{tabular}{|c|c|c|c|}
\hline \multicolumn{3}{|c|}{ PHASE-IV (45 Days later to Phase-I ) N=21 } \\
\hline Method & Result & Number & Percentage \\
\hline \multirow{3}{*}{ Conventional method } & Passed & 18 & $85.7 \%$ \\
\cline { 2 - 4 } & Failed & 03 & $14.3 \%$ \\
\cline { 2 - 4 } & Total & 21 & $100 \%$ \\
\hline \multirow{2}{*}{ DPOAE } & Passed & 20 & $95.2 \%$ \\
\cline { 2 - 4 } & Referred & 01 & $4.8 \%$ \\
\cline { 2 - 4 } & Total & 21 & $100 \%$ \\
\hline
\end{tabular}

Table - VIII: Comparison of Conventional methods with DPOAE at Phase-IV

\begin{tabular}{|l|l|l|l|l|}
\hline \multirow{2}{*}{\multicolumn{2}{|c|}{}} & \multicolumn{2}{|l|}{ Phase-IV. DPOAE } & \multirow{2}{*}{ Total } \\
\cline { 3 - 4 } & Passed & Referred & \\
\hline \multirow{3}{*}{ Conventional methods. } & Passed & 18 & 0 & 18 \\
\cline { 2 - 5 } & $\%$ & $90 \%$ & 0 & $85.7 \%$ \\
\cline { 2 - 5 } & Referred & 02 & 01 & 03 \\
\cline { 2 - 5 } & $\%$ & $10 \%$ & $100 \%$ & $14.3 \%$ \\
\hline \multirow{3}{*}{ Total } & 20 & 01 & 21 \\
\cline { 2 - 5 } & $100 \%$ & $100 \%$ & $100 \%$ \\
\hline
\end{tabular}

Sensitivity $=90 \%$. Specificity $=100 \%$. Positive predictive value $=100 \%$. Negetive predictive value $=33.3 \%$

Screening results of phase-V: In Phase-V, referred for ABR. 1 baby referred to this phase for ABR, have passed this stage.

Table - IX: Screening results of phase-V

\begin{tabular}{|c|c|c|c|}
\hline \multicolumn{4}{|c|}{ Phase $-\mathrm{V}$ ( for ABR) N=1 } \\
\hline Method. & Result & Number & $\%$ \\
\hline ABR & Passed & 1 & 100 \\
\hline
\end{tabular}

\section{Discussion}

The concept of early identification and intervention though not new, is yet to gain a foothold in India. Early detection and management of hearing loss is crucial for the developmental period for auditory, speech, and language acquisition. In recent years, the technology and expertise has developed to allow screening to detect hearing loss in newborn babies. Early detection will also lead to earlier fitting of advanced hearing instruments combined with intensive services targeting communication development.

Screening newborn infants for hearing loss identifies most children with congenital hearing loss prior to the onset of language development, allowing their parents to access services much earlier than otherwise. In the absence of screening, the majority of children with congenital hearing loss do not receive a diagnosis until 2 to 3 years of age, by which point language development is usually seriously delayed. ${ }^{11}$

Otoacoustic Emissions (OAE) Currently acceptable methodology for physiological screening. As it is non invasive, quick and easy to perform .OAE measures sound waves generated in the inner ear in response to clicks or tone bursts emitted and recorded through miniature microphones placed in the external ear canal of the infant. Although OAE screening is quicker and easier to perform, it may be affected by debris or fluid in the external or middle ear resulting in referral rates of 5 to $20 \%$, when screening is performed in the first 24 hours after birth. ${ }^{12}$

The overall pas rate by DPOAE was significantly high at $97.9 \%$. On comparing the Conventional methods with DPOAE, the later showed higher levels of pass rate in early part of life. But as the days advanced the difference between the pass rates between the two methods was reduced. The Specificity (the proportion of 
unaffected babies who are correctly identified by a screening test) and Positive predictive value was significantly high at $100 \%$ for the Conventional method at all the Phases of screening. The Sensitivity (the proportion of babies who are identified by a screening test) of the Conventional methods has progressively increased from Phase-I to Phase-IV (from 20\% to 90\%). Negative predictive value for Conventional methods was high at initial Phase and reduced at later Phases. The drawback of these Conventional methods is that no significant data available to prove that these methods can be used for newborn screening programs.

The results indicate that screening with a portable DPOAE device is a cost effective method which can be easily applied in the neonates to identify infants at risk of hearing loss. The major outcome is the early identification of hearing loss at early part of life which allows early introduction of amplification devices and early intervention to begin. While testing with DPOAE is cheaper in terms of capital equipment cost, we recommend that for a larger program involving all live births automated ABR be used in conjunction with DPOAE as the system of choice. The use of automated ABR technology has the advantages of reduced false positive rate with testing in the first few days of a baby's life, and the ability to detect the $3.5 \%$ of hearing impaired children who have auditory neuropathy. ${ }^{13}$

\section{Conclusion}

Finally timely diagnosis and interventions for hearing loss are not the reality throughout India. The population and healthcare professionals involved in childcare should be made aware of the impact of hearing loss; this could result in increased adhesion to neonatal screening programs.

The results from the present study justifies the use of DPOAE as universal Screening tool to screen the new born babies at early part of life there by helping the infant to cope with early diagnosis and early intervention.

\section{References}

[1]. Watkins P, Baldwin M, Mc Enery G (1991) neonatal at risk screening and the investigation of deafness. Arch Dis Child 66:11301135 .

[2]. Parving A. (1993) Congenital hearing disability: epidemiology and identification: A comparison between two health authority districts. Int J Pediatr Otorhimolaryngo. 27:29-46.

[3]. Auk GW, Behrens TR, (1993) Historical, Political a technological context associated with early identification hearing loss. Seminars in Hearing 14:1:17.

[4]. Northerm JL, Hayes DH, (1994) Universal screening for infant hearing impairment Necessary, beneficial and justifiable. Audilogy Today 6:10-13.

[5]. Kim SY, Bothwell NE, Backousn DD, (2002) The expanding role of the otolaryngologist in managing infants and children with hearing loss. Otolaryngol clin. N Am 35:699.

[6]. Michele AM, De (2004) Newborn hearing screening. eMedicine 1-14.

[7]. White KR, Vohr Br, Behrens TR, (1993) Universal newborn hearing screening using transient evoked otoacoustic emissions: Results of the Rhode Island hearing assessments project. Seminars in Hearing 14:18-29.

[8]. White K, Maxon A, (1995) universal screening for the hearing impairment: simple, beneficial and presently justified. INT J Pediatr Otorhinolaryngol. 32(3):201-211

[9]. Mehl A, Thompson V, (1998) newborn hearing Screening: The Great omission. Pediatrics101:1.

[10]. Isaacson G (2001) Universal Newborn Hearing Screening and Intervention. Advances in Otolaryngology-Head and Neck Surgery 15:1-19.

[11]. Van Naarden K, Decoufle P, Caldwell K. Prevalence and characteristics of Children with serious hearing impairment in metropolitan Atlanta, 1991-1993. Pediatrics 1999;103:570-5.

[12]. Isaacson G (2001) Universal Newborn Hearing Screening and Intervention. Advances in Otolaryngology-Head and Neck Surgery 15:1-19.

[13]. Mehl AL, Thomson V. The Colorado newborn hearing screening project, 1992- 1999: on the threshold of effective populationbased universal newborn hearing screening. Pediatrics, 2002;109 (1):E7. 